\title{
CONTROLLER TEAM POSSIBILITIES FOR SECTORLESS AIR TRAFFIC MANAGEMENT
}

\author{
Bettina Birkmeier, Sebastian Tittel, Bernd Korn \\ German Aerospace Center, DLR, Braunschweig, Germany
}

\begin{abstract}
Sectorless air traffic management is an en-route concept, which eliminates the need for control sectors. Instead of assigning a geographic area (sector), air traffic controllers are assigned certain aircraft. Controllers are responsible for their assigned aircraft all the way from entry to exit.

In previous simulations, one controller was responsible for six aircraft at the same time. As these aircraft can be located anywhere in the sectorless airspace, controllers were provided with one traffic display for each assigned aircraft.
\end{abstract}

This discussion paper suggests other possibilities for providing traffic information to sectorless air traffic controllers. Instead of having one traffic display for each aircraft under control (tiled display), controllers could work with a general map, a zoom display to magnify certain traffic situations, or a combination of such displays.

We revisit the concept of controller teams and explore alternatives. In addition to working alone or in pairs of executive and controller, the sectorless air traffic management concept opens possibilities for innovative teamwork. For example, there could be a team of one coordinator and several executives, or even a control-room team.

This paper gives examples of new display and team ideas and discusses their respective advantages and disadvantages. We argue that the way traffic information is displayed to controllers affects their mental models and working methods. In addition, we provide results on the tiled display from previous simulations and introduce ideas for future research.

\section{Introduction}

Sectorless air traffic management (ATM) has been researched at the German Aerospace Center DLR since 2008 [1]. It is an en-route concept for air traffic control, where controllers are no longer in charge of geographic sectors but are assigned individual aircraft anywhere in the airspace. Controllers are responsible for the assigned aircraft from their entry into the sectorless airspace until their exit.

Previous DLR papers have investigated various aspects of the concept: General feasibility of the concept for upper airspace was proven in 2011 [2]. A 2010 paper discussed the compatibility of sectorless ATM with the Single European Sky ATM Research (SESAR) [3]. The concept will be part of SESAR 2020 as described in the multi-annual work programme [4]. Further DLR research covered priority rules [5], assignment strategies [6], the controller's mental model [7], controller tasks [8], a safety net [9], a safety assessment [14], transition strategies [15], and color schemata for the controller working position [16]. A research report [17] summarizes DLR research on sectorless ATM between 2009 and 2014.

Validations have been run on DLR's TrafficSim, a simulator which is capable of fast-time and realtime simulations [2]. In real-time simulations, the simulator can be equipped with as many controller working positions (CWPs) as needed; traffic which is not assigned to simulation controllers can be guided by the simulator.

Among experts and simulation participants, the design of the CWPs has been controversially discussed. Current CWP design supports a method of working where one controller is responsible for six aircraft at the same time. The decision to have just one sectorless controller instead of a team of executive and planner [7] has been another topic of many discussions [9].

For a general introduction to the concept of teams and teamwork, please refer to Paris et al. [10]. They give a review of team research, including an introduction to teams, team member selection, and team performance and effectiveness assessment. Paris et al. claim that among others, shared situation awareness is one behavior or skill of teams. EUROCONTROL [13] provides more specific 
background on the topic of air traffic controller teams.

For an introduction to a general situation awareness and mental models, please refer to Endsley [11]; EUROCONTROL [12] provides more specific definitions for air traffic control.

In the following, we elaborate on further ideas for controller teams and CWPs in a sectorless working environment. A good combination of controller teams and a suitable support through the displays and systems of the CWP is necessary to optimally support sectorless controllers in their work.

We first introduce sectorless ATM including the controller team and CWP setups that have been used so far. Thereafter, we recap the influence of team and CWP setups on the controller's mental model. We then introduce new ideas for traffic displays and controller teams, before discussing their advantages and disadvantages. A conclusion shows that the suitability of a setup might be dependent on the airspace and other constraints.

\section{The Concept of Sectorless ATM}

In order to balance demand and capacity in enroute ATM, the current practice is to partition the airspace into sectors. Each sector is assigned to a pair of air traffic controllers, a team of executive and planner. This controller team is responsible for the traffic within the sector [18].

When the workload reaches the sector capacity limit, the sector is subdivided into smaller sectors. Each of these smaller sectors is then assigned to responsible air traffic controllers, thus distributing the workload [18].

However, an increase in the number of sectors also increases the amount of necessary coordination between controllers and handoffs from one sector to the next. In addition, the time an aircraft remains in one sector and the maneuvering space becomes smaller. Therefore, sectors can only be subdivided a limited number of times before further division becomes infeasible.

The concept of sectorless ATM provides a solution to this problem by considering the airspace as one piece without subdivisions. Instead, an assignment center assigns individual aircraft to the controllers. Workload can be distributed evenly among the air traffic controllers on duty. One controller is then responsible for a certain number of aircraft anywhere in the airspace and stays in charge all the way from entry to exit.

The current DLR simulation environment provides each controller with one traffic display for each aircraft under control. The controller is only responsible for one aircraft in each display; surrounding traffic is controlled by other sectorless controllers. This way of displaying information to the controller is called a tiled display.

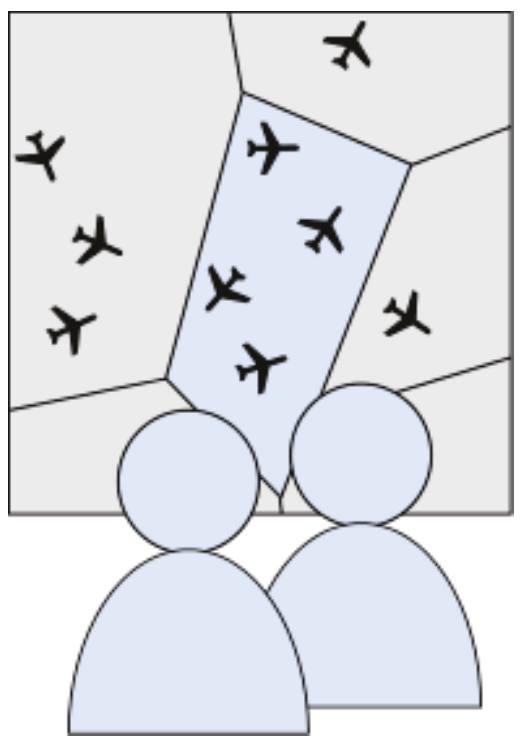

Figure 1. Sectored Setup

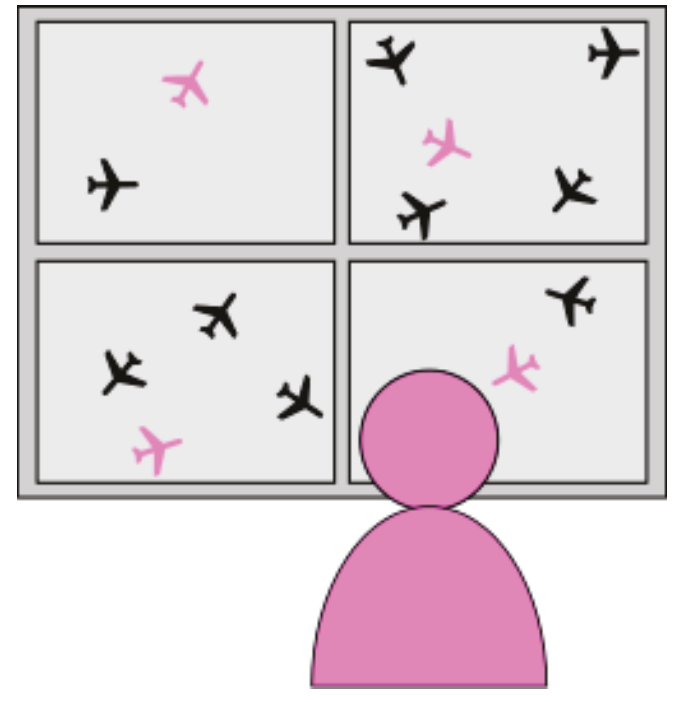

Figure 2. Tiled Display 
Figure 1 shows a sketch of the traditional sectored setup. Two controllers are responsible for one sector and the entire traffic within that area. In contrast, Figure 2 shows what a sectorless setup with a tiled display could look like. The controller is only responsible for assigned aircraft, indicated by a pink color.

\section{Previous Controller Team and Controller Working Position Setups}

In previous DLR simulations, each simulation controller was responsible for up to six aircraft at the same time. These aircraft were located anywhere in the sectorless airspace (in our scenarios the upper airspace over Germany). In order to support their work, controllers were provided one traffic display for each aircraft they had under control.

CWPs consisted of a large screen (56 inches diagonal), which was divided into several information areas, and a computer mouse as input device. Figure 3 shows a picture of the CWP screen as it was used in the validations. The picture is superimposed with white lines to indicate the information areas, in this case six radar screens R1$\mathrm{R} 6$, a conflict list $\mathrm{C}$ and a situation display $\mathrm{S}$.

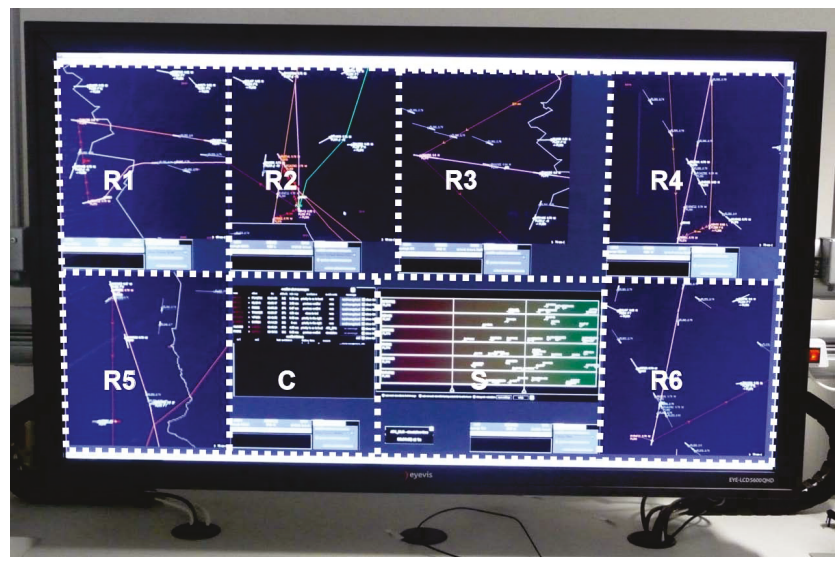

Figure 3. Tiled Display Used in Previous Validations

\section{Influence of Team and CWP Setups on the Controller's Mental Model}

A task analysis [8] and the evaluation of first results on situation awareness and workload [7] in sectorless ATM provided first insights into the change of controller tasks and mental models.

Main results were that in sectorless ATM the tasks of the planner are either not necessary anymore (such as coordination with adjoining sectors), or are taken over by the system. Therefore, Birkmeier et al. [7] stated that there is a shift of tasks: The controller's main tasks shift toward more tactic control and monitoring while support systems take over planning tasks (see Figure 4).

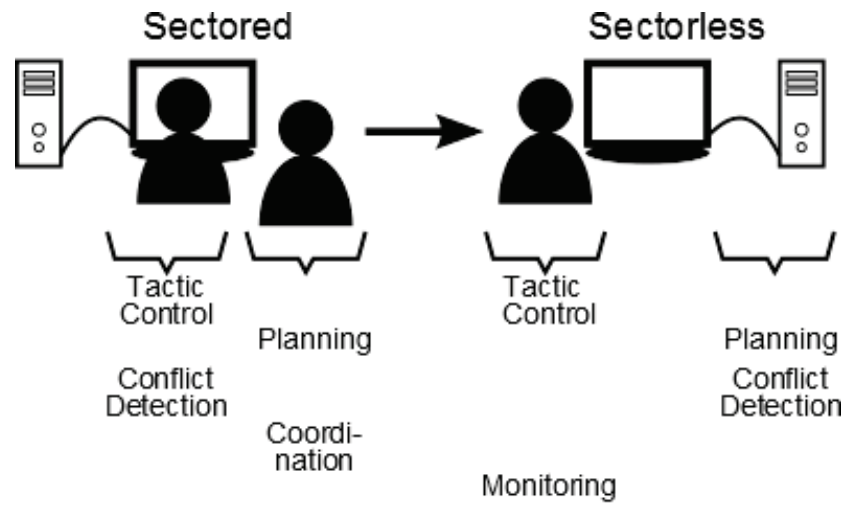

Figure 4. Task Shift

In addition to the task shift, also the mental model of controllers is changed. As described by Birkmeier et al. [7], the "picture" of the controller in a sectored setup is a mental representation of the traffic situation in a certain area (usually the sector), which can be projected into the future for a certain amount of time (see Figure 5).

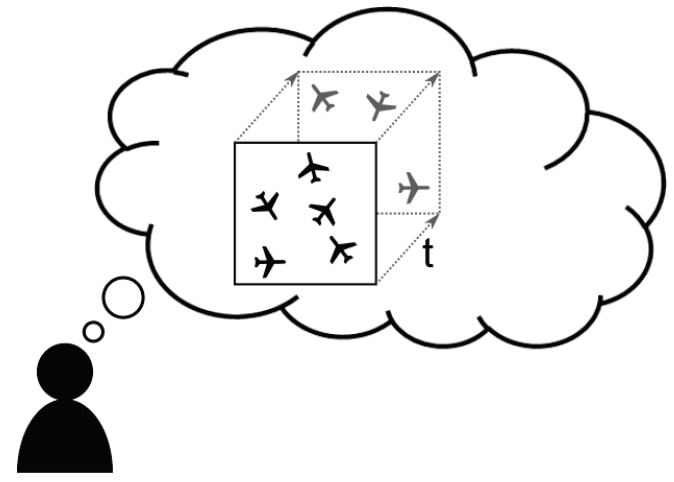

Figure 5. Controller's Mental Model in Sectored ATM

In contrast, in sectorless ATM, first results show that the controller maintains several traffic pictures. Each of these pictures is smaller than in sectored 
ATM, and can be projected into the future by a smaller amount of time (see Figure 6). Nevertheless, controller feedback indicated that in fact, sectorless controllers still maintain situation awareness and pictures of the traffic situations [7].

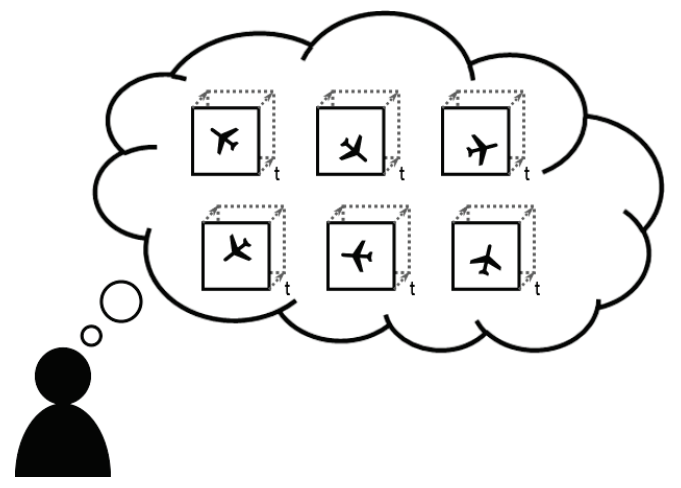

Figure 6. Controller's Mental Model in Sectorless ATM

Of course, the mental model is probably influenced by the way information is provided to the controller. It is likely that the subdivision into smaller pictures arises from the tiled display, where each aircraft is given its own traffic display. Future research should investigate the influence of different types of displays, tools and their layout on the way the controllers work and build up their mental traffic picture. This connection could even be used to define the work, tasks, and procedures in a sectorless ATM concept.

In future simulations, different setups for traffic displays and controller teams should be tested in order to evaluate their influence on the controller work and situation awareness. A thorough investigation of different setups should help to choose the best possible setup for future implementations of sectorless ATM.

\section{New Ideas for Traffic Displays}

When validating a concept, one has to decide on certain parameters from which to start, and for sectorless ATM one of these parameters was choosing a tiled display and having one controller responsible for up to six aircraft. However, other options are possible. Both the team setup and the CWP setup could be modified and sensible combinations should be found, as not every CWP setup provides good support for each team.

\section{Geographic Overview Display}

Instead of having one radar display for each aircraft under control (tiled display), controllers could work with a general overview map. On such a map, each aircraft under control would be marked in order to distinguish it from surrounding traffic.

As it is envisioned that the sectorless airspace will be rather large compared to today's sectors, such a geographic overview display entails that there will be a lot of information to be displayed. Either this means that there will be a lot of information in the display, which might lead to clutter and information excess, or that information will have to be filtered. In any case, it makes sense to provide the controllers with the possibility to choose how much information they want to be displayed, e.g., by letting them choose level bands or other ways of filtering information.

Figure 7 shows a possible geographic overview display. The complete sectorless airspace (in this case Germany) can be seen in one display. Assigned aircraft are color-coded. Strong filtering must apply in order to keep the information on a level that can still be processed by the controller. Still, representations and labels must be very small in order to fit everything on the screen.

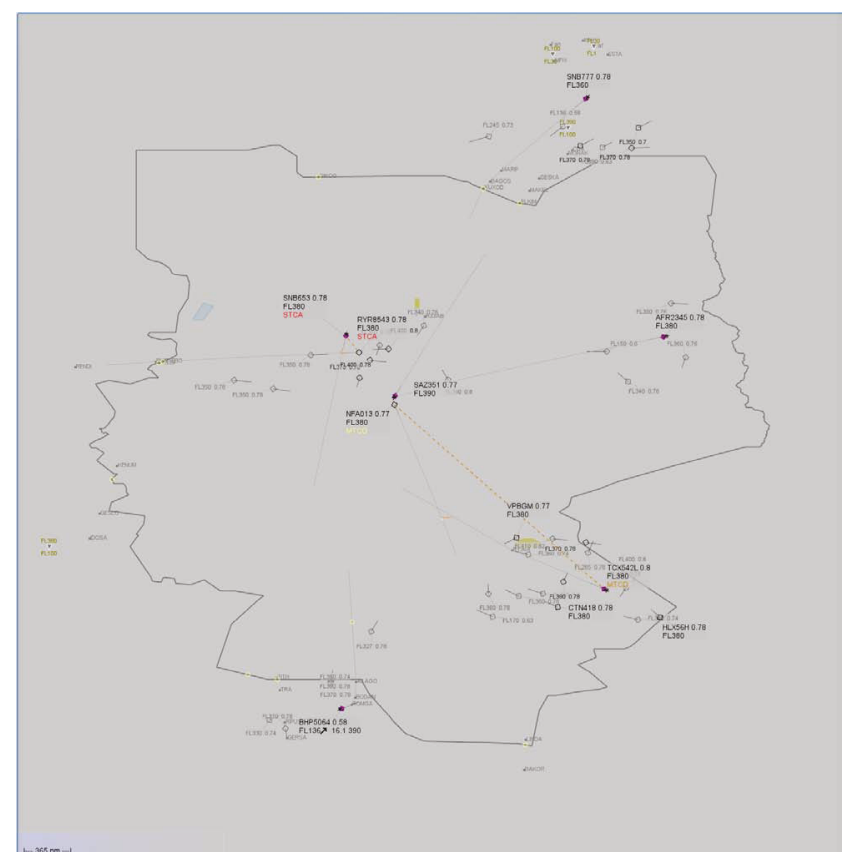

Figure 7. Possible Geographic Overview Display 


\section{Magnifying Display}

As it is hard to display detailed information in overview displays, a tool similar to a magnifying glass could be used. Controllers select a certain area on the geographic overview display to magnify it. This means, not only that information is displayed larger but also that also different filters apply to the information.

Figure 8 shows an example for the design of such a magnifying display. One part of the map is shown in more detail in the blue square superimposed on the geographic map.

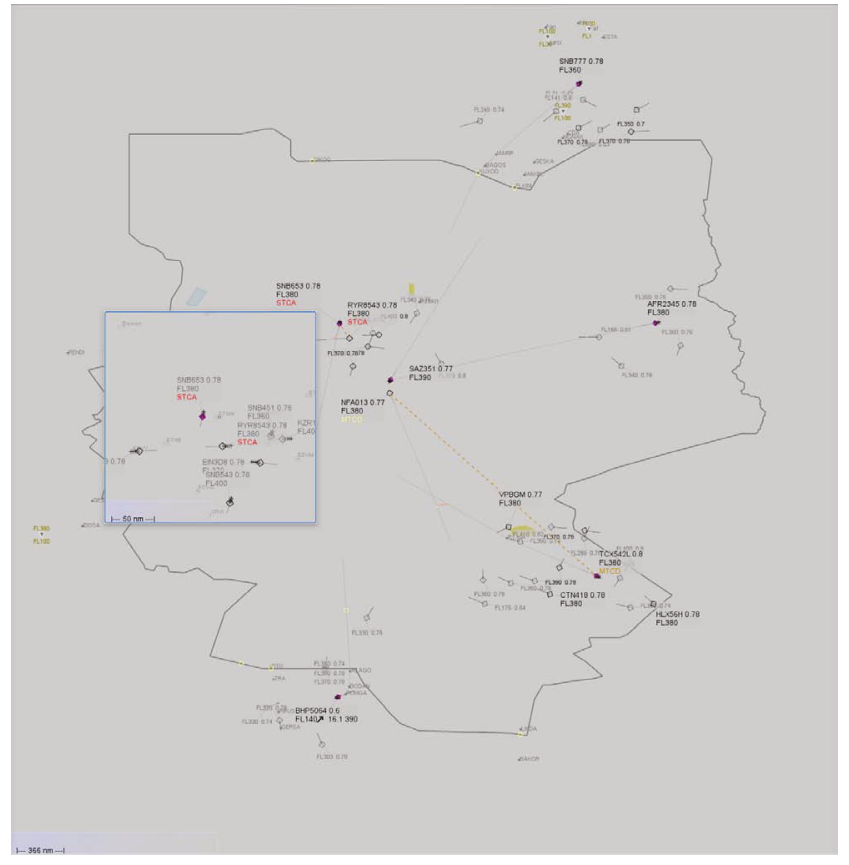

Figure 8. Magnifying Display

\section{Zoom Display}

As the magnifying display obscures part of the geographic display, it might be better to have an additional zoom window. Such a zoom display works similar to the magnifying display but is located beside, above, or below the geographic overview display. It shows a clipping of the overview display, but provides the information larger and with more detail. This could be especially helpful when analyzing conflicts and searching for avoidance maneuvers.

Figure 9 shows an example sketch of such a zoom display. The controller can choose an area on the geographic overview display, which is then magnified in an additional zoom display.

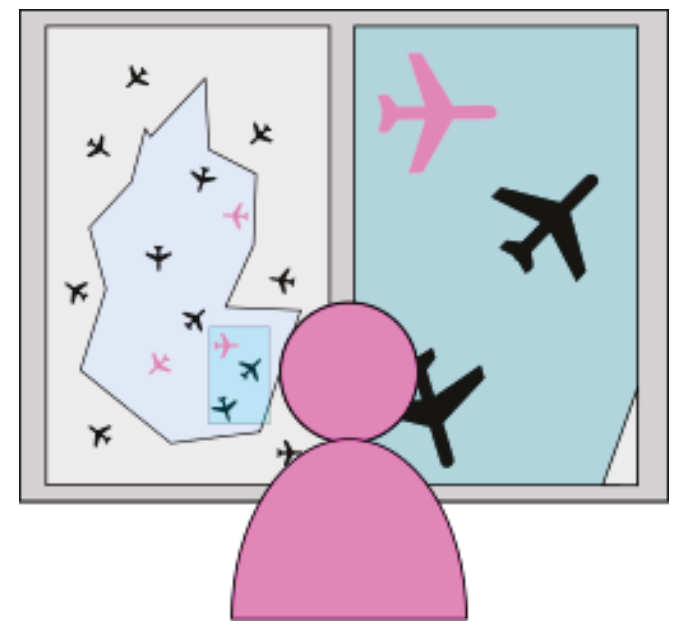

Figure 9. Zoom Display

When combining more than one zoom display with a geographic overview display, one gets a combination of the tiled and the geographic overview display.

Figure 10 shows an example where the geographic overview display in the middle was combined with six zoom displays, three to the left and three to the right of the geographic overview display.

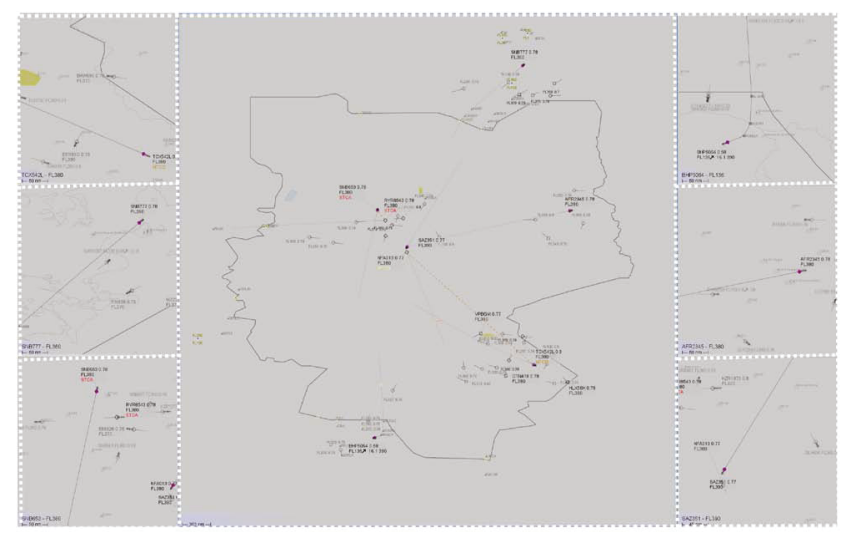

Figure 10. Combination Display

\section{Cinema Display}

One could also imagine a display, which can be shared by several controllers. On a big cinema-like screen, information that is relevant to everybody, e.g., geographic overview, could be displayed while 
each of the controllers retains his own detailed view on an individual screen.

Figure 11 shows an example sketch of such a cinema display. In addition to traffic information the cinema display could show graphs, weather information, analyses, or any other information that is relevant to all controllers. Each controller has individual information on his or her own screen.

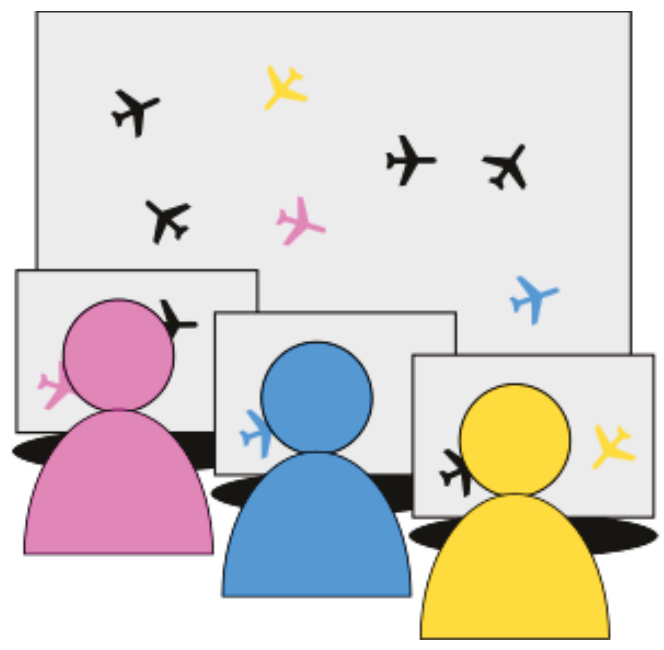

Figure 11. Cinema Display

\section{New Ideas for Controller Teams}

In the validation simulations for sectorless ATM, one controller was responsible for up to six aircraft. The responsibilities of the planner were largely taken over by the systems; the remaining sectorless controller relied on system support for planning and focused on a more tactic control [7]. Like the display setup, this was just a parameter that had to be chosen when designing the validations. Nevertheless, other setups are possible and remain to be tested.

\section{Executive and Planner Team}

One possibility for controller teams is to implement a setup similar to the current setup in sectored ATM. Two controllers could work as a team similar to today's teams of executive and planner. The executive's main task would be to give instructions to pilots by voice communication while the planner is mainly responsible for coordination and developing early resolutions for conflicts.

\section{Two-Controller Team}

Since first validations have shown that the tasks of the planner are mostly taken over by the systems, two controllers could also work together differently. They could be assigned a larger number of aircraft and work on them together. Validations must show what this teamwork could look like, and how workload could be distributed between two sectorless controllers who are jointly in charge of several aircraft.

Figure 12 shows a sketch of two controllers in charge of several aircraft, in this case supported by a tiled display.

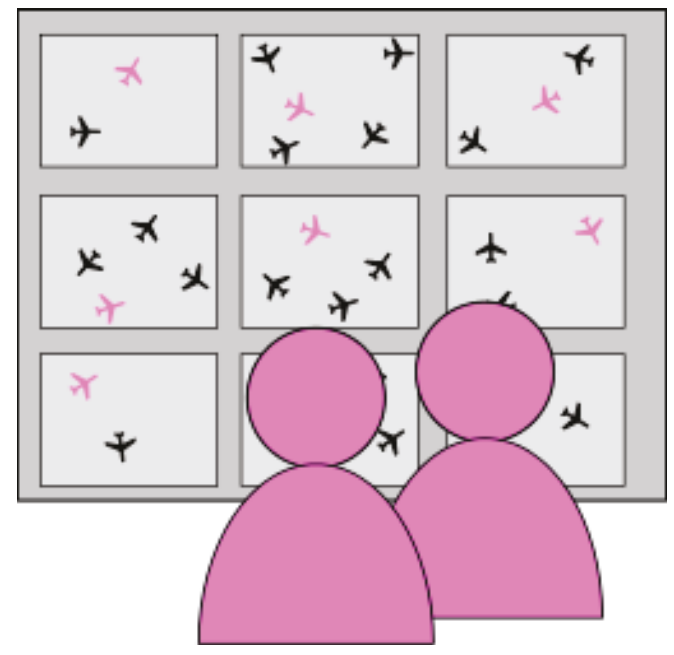

Figure 12. Two-Controller Team

\section{Coordinator-Executives Team}

Similar to the idea of the multi-sector planner [4], one can imagine a team of one coordinator and several executives in a sectorless setup. Such a coordinator could support the other controllers in handoff situations. Coordinators could be part of the assignment center [17].

\section{Control-Room Team}

There could even be a stronger team connection between several sectorless controllers and several coordinators if they work together in one control room. For example, with the support of a cinema display, they could share displays in order to reach shared situation awareness. 


\section{Discussion}

Each of the setup described above has their own advantages and disadvantages, which we discuss in the following.

\section{Tiled Display}

For first simulations the tiled display was chosen because it was straightforward. As aircraft were too far spread for showing them in only one display, clippings of the relevant traffic situations were chosen. The tiled display has the advantage that controllers can monitor all traffic situations at the same time; everything is shown all the time. By choosing the zoom level, controllers can decide on the amount of detail they want to see.

The disadvantage is that there is only a certain amount of tiles that can be displayed even on a large screen. At a certain point the tiles become too small to sensibly hold all relevant information. The screen itself is also limited in size for ergonomic reasons.

Therefore, the tiled display limits the number of aircraft that can be assigned to one sectorless controller. This does not limit capacity in general, as higher traffic demand can be balanced by having more air traffic controllers on duty. Nevertheless, such a limitation is undesirable, as controller efficiency (controlled flight hours per controller hour) should be limited only by the traffic and workload, not by the displays.

\section{Geographic Overview Display}

The geographic overview display simply increases the range of original sectored traffic displays. This has the advantage that geographic overview is given: controllers can easily locate where their assigned aircraft are in the sectorless airspace. However, such a geographic overview might is not strictly necessary anymore in sectorless ATM [9].

Such an overview display has the advantage that there is no limit to the number of aircraft that can be assigned to one controller. Thus, in contrast to the tiled display, the geographic overview display does not limit controller efficiency by design.

However, the display requires heavy filtering and the large range entails that not all details can be displayed. Therefore, the geographic overview display is not well suited for detailed analysis as it is, e.g., necessary in conflict situations. The limited detail of the display might also have a negative effect on situation awareness, which should be investigated in future simulations.

\section{Magnifying Display}

The magnifying display can help overcome the disadvantages of the geographic display. By choosing one area, which is magnified, controllers can regain the level of detail, which is needed for conflict analyses. However, a "magnifying lens" on top of the geographic display obscures the area underneath.

\section{Zoom Display}

Therefore, it makes sense to transfer the magnifying area to an additional display. This retains the advantages of the geographic overview display and the magnifying display while resolving their disadvantages.

Depending on the number of assigned aircraft it might make sense to provide controllers with more than one zoom display so that they can choose a number of areas which they want to see in detail.

\section{Cinema Display}

The cinema display is closely connected to the idea of forming larger teams. The advantage of the large screen is that groups of more than two people can share common information, which is relevant to everybody. This information can be a geographic traffic overview, but could also contain weather or conflict information as well as analytical diagrams.

The disadvantage of the cinema display is that only a limited number of controllers can be placed in such a way that they can comfortably view the cinema display. In addition, they will have to switch between their own information screens and the large screen, which could lead to ergonomic issues.

\section{Single Sectorless Controller}

In previous DLR simulations on sectorless ATM, a single controller was working with up to six assigned aircraft. Task analyses showed that the traditional tasks of the planner could be mostly taken over by support systems such as medium-term conflict detection [7]. 
Such a single operation worked well in the validations so far. Controllers expressed that they are relying heavily on system support [7], which is only to be expected with this kind of automatization. Such a mode of operation and automatization is also the enabler for the expected increase in efficiency [1].

Nevertheless, there are disadvantages of single sectorless controller operations. Even though a foureye-principle can be expected to remain for conflicts [14], there are more advantages to a controller team. Working together in teams could help controllers discuss and solve conflicts more efficiently or stay more alert to critical situations. These anticipated effects should be carefully investigated.

\section{Executive and Planner Team}

One obvious possibility for sectorless controller teams is to retain the current setup of executive and planner. However, since previous results have shown that planner tasks are mostly taken over by the systems [7], the work of the planner must be redefined. For example, the planner could be responsible for a contact to the assignment center.

The advantages of reintroducing a work partner have already been discussed in the section above. Controllers would have a partner to discuss with and to keep aware of the traffic situations. Disadvantages are mainly the as of yet unclear tasks of the sectorless planner and a potential negative effect on controller efficiency as described above.

Disadvantages are mainly the as of yet unclear tasks of the sectorless planner and a potential negative effect on controller efficiency as described above.

\section{Coordinator-Executives Team}

A potentially negative effect on efficiency could be mitigated by introducing a multi-executive coordinator, similar to the idea of a multi-sector planner [4]. One coordinator controller could be working with multiple sectorless executive controllers and support them, e.g., with assignment center contacts and traffic situation awareness.

Expected advantages are similar to the ones expected for executive and planner teams, while keeping the impact on controller efficiency low.

\section{Control-Room Team}

The idea of a control-room team takes the above team ideas even further. Forming such a team would give each controller a number of partners to work with while keeping the impact on efficiency low. It could even be possible to have different tasks in such a control room, i.e., sectorless executive controllers and coordinators. Even the assignment center staff could be located in the same room to stay in direct contact with executives and coordinators.

Advantages and disadvantages of such a setup remain to be carefully investigated. It is expected that such a team setup could combine the advantages of all abovementioned ideas while keeping efficiency at a high level.

A natural support setup for a control-room team would be the cinema display.

\section{Conclusions}

Sectorless ATM has already been proven to deliver huge benefits in en-route capacity and controller efficiency at the same time [17]. However, the design of sectorless controller working positions and consequently the way controllers will work in this new environment will have an effect on controller efficiency and thus on the costs for providing service to airspace users.

The controller working positions tested in previous validations have limitations on how many aircraft can be assigned to one controller at the same time: Either by design as in a tiled display configuration, or by limited situation awareness as it could occur when having too many aircraft under control with a geographic overview display or a zoom display configuration.

Introducing teams in sectorless ATM seems to be a promising way to increase controller efficiency while retaining situation awareness.

Future research on sectorless ATM should include the aspects of controller teams, team organization and with them the aspects of setting up CWPs that support those teams best. This research should take prior research on teamwork into consideration, e.g., Paris et al. [10], or specific prior work on controller teams, e.g., EUROCONTROL [13], or Papenfuss et al. [19], [20]. 
Different teams and setups might be suitable for different airspaces. For example, while a geographic overview display might be perfectly suitable for smaller airspaces, it might be difficult to sensibly fit a larger airspace on one display. Similarly, having a supporting coordination controller might make sense for airspaces where there are many handoffs to lower or adjacent airspaces, while for other airspaces such a coordination controller might be superfluous. Therefore, an analysis of different sectorless setups might be of interest, in order to evaluate which team and CWP setups are most suitable for which airspaces.

As the work progresses, recommendations for sectorless controller teams and CWPs should be made depending on the concerned airspace, procedures, and traffic patterns.

\section{References}

[1] Korn, Bernd, Christiane Edinger, Sebastian Tittel, Dirk Kügler, Thomas Pütz, Oliver Hassa, Bernd Mohrhard, 2009, Sectorless ATM-A Concept to Increase En-Route Efficiency, Proceedings of the 28th Digital Avionics Systems Conference (DASC) 2009, Orlando, FL, USA.

[2] Biella, Marcus, Bettina Birkmeier, Bernd Korn, Christiane Edinger, Sebastian Tittel, Dirk Kügler, 2011, Operational Feasibility of Sectorless ATM, Proceedings of the International Conference of the European Aerospace Societies (CEAS) 2011, Venice, Italy.

[3] Birkmeier, Bettina, Bernd Korn, Dirk Kügler, 2010, Sectorless ATM and Advanced SESAR Concepts: Complement not Contradiction, Proceedings of the 29th Digital Avionics Systems Conference (DASC) 2010, Salt Lake City, UT, USA.

[4] SESAR Joint Undertaking, 2015, SESAR 2020 Multi-Annual Work Programme, Version 1.0.

[5] Birkmeier, Bettina, Christiane Edinger, Sebastian Tittel, Bernd Korn, Dirk Kügler, 2011, First Results on Flight Rules and Conflict Avoidance Maneuvers for a Sectorless ATM Concept, Proceedings of the 11th Integrated Communications Navigation and Surveillance Conference (ICNS) 2011, Herndon, VA, USA.

[6] Schmitt, Angela Rebecca, Christiane Edinger, Bernd Korn, 2011, Balancing Controller Workload
Within a Sectorless ATM Concept, CEAS Aeronautical Journal, 2:35-41.

[7] Birkmeier, Bettina, Bernd Korn, Frank Ole Flemisch, 2011, First Findings on the Controller's Mental Model in Sectorless Air Traffic Management, Proceedings of the 30th Digital Avionics Systems Conference (DASC) 2011, Seattle, WA, USA.

[8] Birkmeier, Bettina, Julia Schmid, Angela Rebecca Schmitt, Bernd Korn, 2012, Change of Controller Tasks in a Sectorless ATM Concept First Results, Proceedings of the 12th Integrated Communications Navigation and Surveillance Conference (ICNS) 2012, Herndon, VA, USA.

[9] Birkmeier, Bettina, Marcus Biella, 2012, Safety Net for a Sectorless Air Traffic Management Concept, Proceedings of the 31st Digital Avionics Systems Conference (DASC) 2012, Williamsburg, VA, USA.

[10] Paris, Carol, Eduardo Salas, Janis CannonBowers, 2000, Teamwork in Multi-Person Systems: A Review and Analysis, Ergonomics, 43(8), 10521075.

[11] Endsley, Mica, 1995, Toward a theory of situation awareness in dynamic systems, Human Factors, 37(1), 32-64.

[12] Kallus, Wolfgang, Manfred Barbarino, Dominique van Damme, 1997, Model of the Cognitive Aspects of Air Traffic Control, European Air Traffic Control Harmonization and Integration Programme (EATCHIP), Eurocontrol, first edition.

[13] Mellet, Uwe, Antonio Licu, 2009, Team Coordination Study, Eurocontrol, first edition.

[14] Birkmeier, Bettina, David Martín, Karsten Straube, Marcus Biella, 2013, Preliminary Safety Assessment for a Sectorless ATM Concept, Proceedings of the 32nd Digital Avionics Systems Conference (DASC) 2013, Syracuse, NY, USA.

[15] Birkmeier, Bettina, Bernd Korn, 2014, Five Transition Strategies for Sectorless ATM, Proceedings of the 33rd Digital Avionics Systems Conference (DASC) 2014, Colorado Springs, CO, USA.

[16] Birkmeier, Bettina, Daniel Diethei, Karsten Straube, Marcus Biella, Sebastian Tittel, 2015, Color Schemes for a Sectorless ATM Controller Working Position, Proceedings of the 34th Digital Avionics 
Systems Conference (DASC) 2015, Prague, Czech Republic.

[17] Birkmeier, Bettina, 2015, Feasibility Analysis of Sectorless and Partially Automated Air Traffic Management, $\mathrm{PhD}$ Dissertation as DLR Forschungsbericht 2015-12, ISSN 1434-8454.

[18] Baumgartner, Marc, 2007, The Organization and Operation of European Airspace, in Andrew Cook, editor, European Air Traffic Management: Principles, Practice and Research, pages 1-34, Ashgate Publishing Lt., Aldershot, UK.

[19] Papenfuss, Anne, Christoph Möhlenbrink, 2011, The Opportunities and Challenges of Team Work in Remote Tower Operations, presented at the 9th Berliner Werkstatt Mensch-Maschine-Systeme, Berlin, Germany.
[20] Papenfuss, Anne, 2013, Phenotypes of Teamwork - an Explorative Study of Tower Controller Teams, Proceedings of the Human Factors and Ergonomics Society Annual Meeting 2013, 57:319-323.

\section{Email Addresses}

Bettina.Birkmeier@dlr.de

Sebastian.Tittel@dlr.de

Bernd.Korn@dlr.de

2016 Integrated Communications Navigation and Surveillance (ICNS) Conference

April 19-21, 2016 